\title{
O USO DE TECNOLOGIAS EDUCACIONAIS PARA IDOSOS: UMA REVISÃO INTEGRATIVA DA LITERATURA
}

\section{THE USE OF EDUCATIONAL TECHNOLOGIES FOR THE ELDERLY: AN INTEGRATIVE LITERATURE REVIEW}

\author{
Gustavo de Souza Lira ${ }^{1} *$ Maria Rafaela Dias de Freitas ${ }^{2} *$ Bruno Freire Braun $^{*}$ \\ Chaves $^{3} *$ Alêssa Cristina Meireles de Brito ${ }^{4} *$ Fabiana Ferraz Queiroga Freitas $^{5}$
}

\begin{abstract}
RESUMO
O envelhecimento é um processo contínuo marcado por alterações físicas e biológicas. Diante essa realidade, as tecnologias educativas (TE) configuram-se como oportunidade de trabalhar a educação de forma mais dinâmica e atrativa. Objetivou-se analisar a produção científica abordando as tecnologias educacionais desenvolvidas e/ou validades para o cuidado com a pessoa idosa. Trata-se de uma revisão integrativa de literatura, método que possibilita a síntese do estado de conhecimento e conclusões gerais sobre uma determinada temática a partir da análise de vários estudos publicados. Assim, analisou-se a importância da abordagem avaliativa afim de avaliar os declínios inerentes ao ser idoso tendo a implementação de programas de promoção à saúde melhora a vida e saúde. Sugere-se o desenvolvimento de outros estudos que desenvolvam e validem tecnologias educacionais para pessoas idosas capazes de estimular os domínios da cognição, humor, mobilidade e comunicação.
\end{abstract}

Palavras chaves: Tecnologia Educacional; Idoso; Envelhecimento; Estudos de Validação; Cognição.

\begin{abstract}
Aging is a continuous process marked by physical and biological changes. Given this reality, educational technologies (TE) are configured as an opportunity to work on education in a more dynamic and attractive way. The objective was to analyze scientific production addressing the educational technologies developed and/or validities for the care of the elderly. It is an integrative literature review, a method that allows the synthesis of the state of knowledge and general conclusions on a given theme from the analysis of several published studies. Thus, the importance of the evaluative approach was analyzed in order to assess the inherent declines in the elderly, having the implementation of health promotion programs improves life and health. It is suggested the development of other studies that develop and validate educational technologies for elderly people capable of stimulating the domains of cognition, humor, mobility and communication.
\end{abstract}

Keywords: Educational Technology, Aged, Aging, Validation Studies; Cognition.

\footnotetext{
${ }^{1}$ Graduando em Enfermagem pela Universidade Federal de Campina Grande. Membro da linha de pesquisa Saúde do Idoso do Laboratório de Tecnologias de Informação e Comunicação em Saúde - LATICS. Cajazeiras, Paraíba, Brasil.

${ }^{2}$ Graduanda em Enfermagem pela Universidade Federal de Campina Grande. Membro da linha de pesquisa Saúde do Idoso do Laboratório de Tecnologias de Informação e Comunicação em Saúde - LATICS. Cajazeiras, Paraíba, Brasil.

${ }^{3}$ Graduando em Enfermagem pela Universidade Federal de Campina Grande. Membro da linha de pesquisa Saúde do Idoso do Laboratório de Tecnologias de Informação e Comunicação em Saúde - LATICS. Cajazeiras, Paraíba, Brasil.

${ }^{4}$ Enfermeira. Pós-graduada em Docência e Pesquisa na Área da Saúde pela Instituto Educacional Maris. Graduada em Enfermagem pela Universidade Federal de Campina Grande. Membro da linha de pesquisa Saúde do Idoso do Laboratório de Tecnologias de Informação e Comunicação em Saúde - LATICS. Cajazeiras, Paraíba, Brasil.

${ }^{5}$ Enfermeira. Doutora em Enfermagem pela Universidade Federal de Minas Gerais. Docente da Universidade Federal de Campina Grande. Líder do Grupo de Pesquisa Laboratório de Tecnologias de Informação e Comunicação em Saúde - LATICS. Cajazeiras, Paraíba. Brasil.
} 


\section{INTRODUÇÃO}

O envelhecimento é um processo contínuo marcado por alterações físicas e biológicas inerentes a todos os seres humanos, sendo vivenciado de forma singular por cada indivíduo. Trata-se de uma fase natural da vida, que deve ser encarada como tal.

No Brasil, desde a década de 60, o envelhecimento vem se tornando um fenômeno que tende a aumentar cada vez mais, em decorrência do aumento de expectativa de vida. Diferente de outros países, no entanto, o Brasil é considerado um país jovem, com cerca de 9,1\% (52 milhões) de população com idade acima de 60 anos ${ }^{(1-}$ 2).

Dentre as diversas alterações que o envelhecimento pode vir a acarretar no corpo humano, a diminuição da capacidade cognitiva é um dos principais alvos de queixas, especialmente associada ao esquecimento. As alterações cognitivas frequentemente observadas, incluem a dificuldade em realizar atividades motoras, tratar e manipular informações visuais e espaciais, executar duas tarefas ao mesmo tempo, esquecimentos de fatos recentes, entre outros, o que pode acarretar em diversos sentimentos negativos por parte da pessoa afetada ${ }^{(3-4)}$.

Diante essa realidade, as tecnologias educativas (TE), caracterizadas por um conjunto de ferramentas sistemáticas com o intuito de preparar, executar e analisar o processo de aprendizagem a fim de torná-lo mais eficaz (5-6), configura-se como oportunidade de trabalhar a educação de forma mais dinâmica e atrativa à pessoa idosa, ao mesmo tempo que pode ser considerada eficaz ao estímulo de funções inerentes ao ser humano, como a cognição.

Essa dinamicidade e atratividade podem ser observadas por meio de técnicas integrativas e participativas, com o objetivo de envolver os sujeitos de forma mais intrínseca no que se refere à construção do conhecimento trabalhado, com clareza e simplicidade ${ }^{(7)}$.

Logo, a efetivação de TE com pessoas idosas, requer um pensamento crítico e reflexivo que requer discussões no âmbito do cuidar em saúde, conjecturando a adoção de ações que viabilizem um olhar atento de profissionais da saúde, para aplicabilidade dessas ferramentas.

Dessa forma, acredita-se que investigar o uso de TE no cuidar de idosos seja relevante por tratar-se de um tema pouco explorado por pesquisadores e profissionais da saúde, premissa evidenciada pelo baixo número de publicações nacionais e internacionais. Objetivou-se analisar a produção científica abordando as tecnologias educacionais desenvolvidas e/ou validades para o cuidado com a pessoa idosa.

\section{MÉTODO}


Trata-se de uma revisão integrativa de literatura, realizada em setembro de 2019, método que possibilita a síntese do estado de conhecimento e conclusões gerais sobre uma determinada temática a partir da análise de vários estudos publicados, podendo apontar também lacunas sobre o conhecimento da temática que ainda precisam ser preenchidas com novos estudos ${ }^{(8)}$.

A elaboração deste método se deu a partir do levantamento bibliográfico pautado nas seguintes fases: 1) definição do tema e seleção da questão de pesquisa; 2) estabelecimento de critérios de inclusão e exclusão; 3) definição das informações a serem extraídas dos estudos selecionados; 4) avaliação dos estudos incluídos na revisão; 5) análise e interpretação dos resultados e 6) apresentação da revisão/síntese do conhecimento ${ }^{(8)}$.

Foi definida a questão norteadora: "quais as tecnologias educacionais e/ou estudos de validação produzidos de 2015 a 2019 sobre o cuidado à pessoa idosa?” Optouse por este período, com o objetivo de identificar e priorizar estudos mais recentes relacionados à temática.

Para atingir o objetivo proposto, foi realizado um levantamento bibliográfico contendo a temática escolhida, utilizando o cruzamento dos seguintes descritores: "tecnologia educacional" AND "idoso" AND "envelhecimento" AND "estudos de validação" AND "cognição". Ademais, utilizou-se filtros relacionados à temática, tais como: cognição, transtornos cognitivos e envelhecimento.

As buscas foram efetivadas no Scientific Electronic Library Online (SciELO) e na Biblioteca Virtual de Saúde (BVS BRASIL) nas bases de dados, Medical Literature Analysis and Retrieval System Online (MEDLINE), Base de Dados de Enfermagem (BDENF) e Literatura LatinoAmericana e do Caribe em Ciências da Saúde (LILACS).

Foram considerados elegíveis artigos disponíveis na íntegra e de forma gratuita, publicados em língua portuguesa, inglesa, espanhola ou alemã entre os anos de 2015 e 2019, sendo excluídos teses, dissertações, monografias, livros, revisões de qualquer estilo e artigos que não abordassem o tema da pesquisa ou que não respondessem à questão norteadora.

Durante a pesquisa no Scientific Electronic Library Online (SciELO) e Base de Dados de Enfermagem (BDENF) não foi possível encontrar nenhum estudo em conformidade aos critérios e cruzamento dos descritores estabelecidos, como também não foi possível produções nas línguas espanhola e alemã, em nenhuma das bases. Nas bases de dados MEDLINE e LILACS foram encontrados 52 artigos. Visando garantir o rigor com a seleção desses artigos, todos os autores participaram desse processo. Os resumos dos 52 artigos foram lidos e, destes, 
31 foram excluídos por não se adequarem à questão norteadora e 5 por serem duplicados (encontrados em mais de uma base consultada, permanecendo apenas em uma base), conforme fluxograma da Figura 1.

\section{Figura 1: Fluxograma representativo da busca nas bases de dados Medline e Lilacs.}

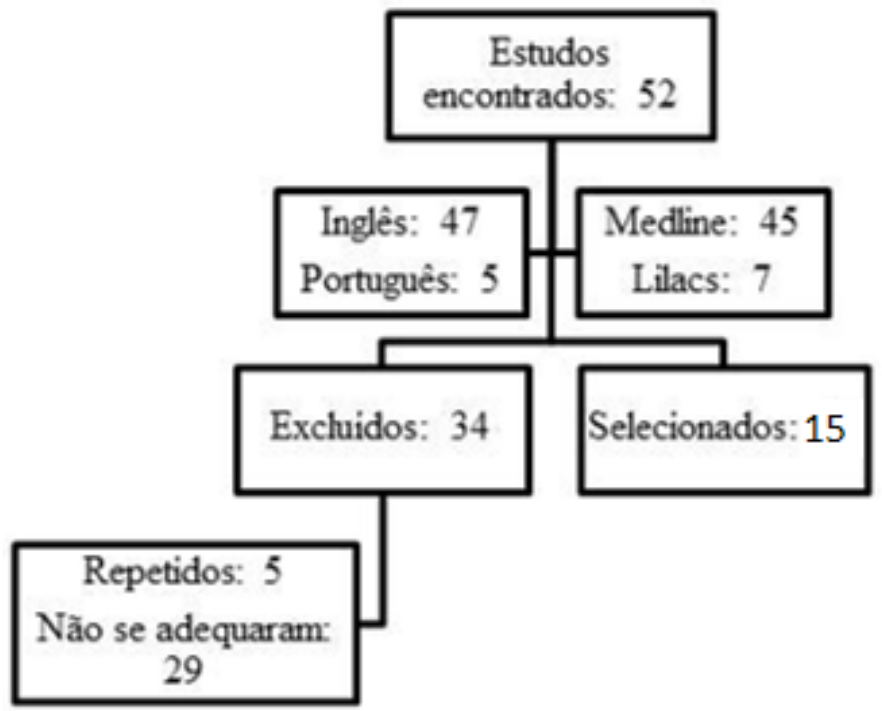

Fonte: Os autores.

Para avaliação na íntegra, foram selecionados 15 artigos, que após caracterização, que considerou as variáveis título, objetivo, tecnologia/inovação e desfecho dos artigos, estabeleceram-se 3 categorias, por similaridade de conteúdo: "ações cuidativas educacionais", "instrumentos avaliativos que auxiliam na compreensão dos declínios da terceira idade" e "a tecnologia cuidativo-educacional e a enfermagem".

\section{RESULTADOS}

Os resultados encontrados foram organizados de maneira sintetizada no Quadro 1, de modo a descrever as principais informações encontradas nas publicações e as categorias que originaram.

Quadro. 1 Apresentação da relação de temas e publicações e síntese dos estudos apresentados na Revisão Integrativa.

\section{Ações cuidativas educacionais}

\begin{tabular}{|l|c|c|c|c}
\hline 1 & $\begin{array}{c}\text { Szturm, T; } \\
\text { Sakhalkar, V; }\end{array}$ & $\begin{array}{c}\text { Integrated testing of } \\
\text { standing balance and }\end{array}$ & $\begin{array}{c}\text { O objetivo deste estudo } \\
\text { foi estabelecer a }\end{array}$ & $\begin{array}{c}\text { Criação de uma plataforma } \\
\text { baseada em jogos de dupla }\end{array}$ \\
\hline
\end{tabular}




\begin{tabular}{|c|c|c|c|c|}
\hline & $\begin{array}{c}\text { Boreskie, S; } \\
\text { Marotta, JJ; Wu, } \\
\text { C; Kanitkar, A. }\end{array}$ & $\begin{array}{l}\text { cognition: Test-retest } \\
\text { reliability and } \\
\text { construct validity }\end{array}$ & $\begin{array}{l}\text { confiabilidade teste- } \\
\text { reteste e construir } \\
\text { validade de uma } \\
\text { plataforma baseada em } \\
\text { jogos de dupla tarefa } \\
\text { (TGP) que integra } \\
\text { rastreamento da cabeça e } \\
\text { tarefas com atividades de } \\
\text { equilíbrio. }\end{array}$ & $\begin{array}{l}\text { tarefa. } \\
\text { A ferramenta de avaliação } \\
\text { amplia outras ferramentas de } \\
\text { teste fornecendo uma } \\
\text { abordagem integrada para a } \\
\text { avaliação do equilíbrio, } \\
\text { estabilidade do olhar e } \\
\text { funções cognitivas executivas } \\
\text { específicas. Análises } \\
\text { misturadas de equilíbrio, olhar } \\
\text { e cognição contribuirão para } \\
\text { uma melhor compreensão de } \\
\text { consequências funcionais do } \\
\text { declínio nas habilidades } \\
\text { físicas e mentais que ocorre } \\
\text { com a idade e nos estágios } \\
\text { iniciais de doenças. }\end{array}$ \\
\hline 2 & $\begin{array}{c}\text { Caprara, M; } \\
\text { Fernández- } \\
\text { Ballesteros, R; } \\
\text { Alessandri, G. }\end{array}$ & $\begin{array}{l}\text { Promoting aging well: } \\
\text { evaluation of Vital- } \\
\text { Aging-Multimedia } \\
\text { Program in Madrid, } \\
\text { Spain (2016) }\end{array}$ & $\begin{array}{c}\text { O Vital Aging- } \\
\text { Multimedia tem como } \\
\text { objetivo promover estilos } \\
\text { de vida saudáveis, } \\
\text { fornecer estratégias para } \\
\text { compensar o declínio } \\
\text { cognitivo e otimizar as } \\
\text { competências afetivas e } \\
\text { sociais e a participação. O } \\
\text { objetivo deste estudo foi } \\
\text { avaliar a eficácia do } \\
\text { programa, empregando } \\
\text { um projeto pré-pós- } \\
\text { intervenção com um } \\
\text { tratamento e um grupo de } \\
\text { controle e enfocando os } \\
\text { quatro principais } \\
\text { domínios de experiência } \\
\text { comumente associados ao } \\
\text { envelhecimento. }\end{array}$ & $\begin{array}{l}\text { Vital Aging-Multimedia. } \\
\text { Após a implementação do } \\
\text { programa VA-M, os } \\
\text { participantes relataram melhor } \\
\text { saúde; melhor memória; } \\
\text { maior frequência de } \\
\text { atividades culturais, } \\
\text { intelectuais e sociais; maior } \\
\text { exercício físico e dietas mais } \\
\text { saudáveis. Além disso, eles } \\
\text { experimentaram menos } \\
\text { emoções negativas visto que } \\
\text { os positivos prevaleceram } \\
\text { sobre os negativos. Estes } \\
\text { achados contrastam com as } \\
\text { ideias e estereótipos dos } \\
\text { idosos como sendo incapazes } \\
\text { de aprender e mudar. }\end{array}$ \\
\hline
\end{tabular}




\begin{tabular}{|c|c|c|c|c|}
\hline 3 & $\begin{array}{c}\text { Williams, KN; } \\
\text { Perkhounkov, } \\
\text { Y; Herman, R; } \\
\text { Bossen, A }\end{array}$ & $\begin{array}{c}\text { A Communication } \\
\text { Intervention to } \\
\text { Reduce Resistiveness } \\
\text { in Dementia Care: A } \\
\text { Cluster Randomized } \\
\text { Controlled Trial } \\
\text { (2017) }\end{array}$ & $\begin{array}{c}\text { Avaliar se uma } \\
\text { intervenção para } \\
\text { melhorar a comunicação } \\
\text { da equipe do lar de idosos } \\
\text { reduzindo o elderspeak } \\
\text { reduziria a resistência ao } \\
\text { cuidado em idosos com } \\
\text { demência. }\end{array}$ & $\begin{array}{c}\text { The Changing Talk - } \\
\text { CHAT/Mudança na forma de } \\
\text { comunicação. } \\
\text { Observou-se que fazer uso de } \\
\text { uma linguagem simples com } \\
\text { os idosos, porém não } \\
\text { infantilizando o indivíduo ou } \\
\text { a situação, ajudou na redução } \\
\text { da resistência ao cuidado. O } \\
\text { estudo comprova a } \\
\text { importância da comunicação } \\
\text { como tecnologia de cuidado, } \\
\text { embora a resistência não tenha } \\
\text { sido eliminada } \\
\text { completamente. }\end{array}$ \\
\hline 4 & $\begin{array}{c}\text { Denny, MC; } \\
\text { Vahidy F; Vu, } \\
\text { KYT; Sharrief, } \\
\text { AZ; Savitz, SI. }\end{array}$ & $\begin{array}{c}\text { Video-based } \\
\text { educational } \\
\text { intervention } \\
\text { associated with } \\
\text { improved stroke } \\
\text { literacy, self-efficacy, } \\
\text { and patient } \\
\text { satisfaction (2017) }\end{array}$ & $\begin{array}{l}\text { - Avaliar a viabilidade da } \\
\text { implementação de } \\
\text { intervenções educacionais } \\
\text { baseadas em vídeo para } \\
\text { pacientes com acidente } \\
\text { vascular cerebral em } \\
\text { hospital; } \\
\text { - Avaliar o grau de } \\
\text { conhecimento inicial dos } \\
\text { pacientes com AVC e; } \\
\text { - Avaliar o impacto do } \\
\text { vídeo no conhecimento } \\
\text { sobre AVC do paciente, } \\
\text { autoeficácia no } \\
\text { reconhecimento dos } \\
\text { sintomas do AVC e sua } \\
\text { influência na educação. }\end{array}$ & $\begin{array}{c}\text { Vídeo educacional. } \\
\text { A maioria dos pacientes } \\
\text { relatou ser capaz de identificar } \\
\text { sintomas da doença após } \\
\text { assistir o vídeo e percebeu-se } \\
\text { satisfação quanto ao mesmo } \\
\text { imediatamente após assistir e } \\
30 \text { dias depois. Enfatiza-se a } \\
\text { importância da educação em } \\
\text { saúde para o público ocorrer } \\
\text { de maneira efetiva, para que } \\
\text { sejam capazes de melhorar o } \\
\text { seu autocuidado, bem como } \\
\text { realizarem autoprevenção. }\end{array}$ \\
\hline 5 & $\begin{array}{c}\text { Dallimore, RK; } \\
\text { Marxengel, } \\
\text { LAT; Chan, D; } \\
\text { Hussain, S; } \\
\text { Willett, C; }\end{array}$ & $\begin{array}{c}\text { A randomised, } \\
\text { double-blinded } \\
\text { clinical study on the } \\
\text { efficacy of } \\
\text { multimedia }\end{array}$ & $\begin{array}{l}\text { Determinar se a educação } \\
\text { do paciente em } \\
\text { fisioterapia, fornecida por } \\
\text { meio de aplicativos do } \\
\text { iPad, foi eficaz em obter }\end{array}$ & $\begin{array}{l}\text { Uso de iPad/Livreto. } \\
\text { Após as intervenções, } \\
\text { pacientes dos } 2 \text { grupos } \\
\text { melhoraram seu nível de } \\
\text { recordação/assimilação a }\end{array}$ \\
\hline
\end{tabular}




\begin{tabular}{|c|c|c|c|c|}
\hline & Zainuldin, R. & $\begin{array}{l}\text { presentation using an } \\
\text { iPad for patient } \\
\text { education of } \\
\text { postoperative hip } \\
\text { surgery patients in a } \\
\text { public hospital in } \\
\text { Singapore (2017) }\end{array}$ & $\begin{array}{c}\text { maior recordação e } \\
\text { satisfação do paciente em } \\
\text { comparação à educação } \\
\text { do paciente fornecida por } \\
\text { meio de um livreto em } \\
\text { papel, em uma coorte de } \\
\text { pacientes submetidos à } \\
\text { cirurgia de quadril. }\end{array}$ & $\begin{array}{l}\text { respeito do que foi mostrado, } \\
\text { além de demonstrar } \\
\text { satisfação. No entanto, isso foi } \\
\text { maior no grupo A (iPad) que } \\
\text { no grupo B (livreto). Assim, } \\
\text { embora ambos métodos } \\
\text { tenham sido eficazes, o uso de } \\
\text { iPad foi considerado mais } \\
\text { efetivo para a educação dos } \\
\text { pacientes em fisioterapia. }\end{array}$ \\
\hline 6 & Li, DM; Li, XX. & $\begin{array}{l}\text { The effect of folk } \\
\text { recreation program in } \\
\text { improving symptoms: } \\
\text { a study of Chinese } \\
\text { elder dementia } \\
\text { patients (2017) }\end{array}$ & $\begin{array}{c}\text { Avaliar os efeitos de um } \\
\text { programa de recreação } \\
\text { popular nos sintomas de } \\
\text { pessoas com demência. O } \\
\text { programa foi adaptado ao } \\
\text { interesse dos } \\
\text { participantes e derivado } \\
\text { de sua cultura tradicional. }\end{array}$ & $\begin{array}{c}\text { Atividades recreativas. Um } \\
\text { programa de recreação } \\
\text { popular com potencial de } \\
\text { melhora da função cognitiva, } \\
\text { da capacidade de vida diária e } \\
\text { os sintomas comportamentais } \\
\text { e psicológicos dos idosos com } \\
\text { demência. As atividades de } \\
\text { lazer folclórico, se inserem no } \\
\text { background cultural dos } \\
\text { participantes, motivando } \\
\text { sentimentos e memórias } \\
\text { positivas, podendo retardar a } \\
\text { progressão da doença e } \\
\text { melhorar os sintomas. }\end{array}$ \\
\hline 7 & $\begin{array}{l}\text { Golino, MTS; } \\
\text { Schelini, PW; } \\
\text { Golino, HF; } \\
\text { Pereira, BLS; } \\
\text { Felix, LM. }\end{array}$ & $\begin{array}{c}\text { Investigando } \\
\text { Evidências de } \\
\text { Validade de Conteúdo } \\
\text { e Estrutural em } \\
\text { Tarefas de um Treino } \\
\text { Cognitivo para Idosos } \\
\text { (2017) }\end{array}$ & $\begin{array}{c}\text { O artigo objetiva reportar } \\
\text { os resultados de dois } \\
\text { estudos de validade } \\
\text { conduzidos em um treino } \\
\text { cognitivo para idosos. }\end{array}$ & $\begin{array}{c}\text { Treino Cognitivo. } \\
\text { De acordo com o modelo } \\
\text { fatorial revelado, há } \\
\text { evidências suportando que o } \\
\text { treino cognitivo proposto } \\
\text { alcançou estimulação } \\
\text { predominante para as } \\
\text { habilidades pretendidas pelo } \\
\text { modelo teórico, porém, com } \\
\text { uma concordância parcial das } \\
\text { tarefas que compuseram cada } \\
\text { domínio. }\end{array}$ \\
\hline
\end{tabular}




\section{8}

Cardoso, RSS.

Sá, SPC;

Domingos, AM;

Sabóia, VM;

Maia, TN;

Padilha, JMFO;

Nogueira, GA.
Tecnologia

educacional: um

instrumento

dinamizador do

cuidado com idosos

(2018)
Desenvolver tecnologia educacional com cuidadores de idosos a partir de necessidades, dificuldades e interesses manifestados por esses indivíduos quanto ao cuidado com a pessoa idosa.
Desenvolvimento de material impresso e digital como auxílio para orientação e informação do cuidado com o idoso.

Desenvolvido, junto aos cuidadores, as tecnologias

educacionais, material impresso e digital, contribuiu para orientação e informação do cuidado com o idoso e para a tomada de decisão do cuidador. Podendo ser utilizado pelo cuidador, população e enfermeiro, mediando a prática educativa com cuidadores.

\section{Instrumentos avaliativos que auxiliam na compreensão dos declínios da terceira idade}

\begin{tabular}{|c|c|c|c|c|}
\hline 9 & $\begin{array}{l}\text { Cornelie, DA.; } \\
\text { Scharloo, M; } \\
\text { Ramondt, S; } \\
\text { Tiemensma, J; } \\
\text { Husson, O; } \\
\text { Llahana, S; } \\
\text { Alberto, MP; } \\
\text { Kaptein, ADA; } \\
\text { Kamminga, } \\
\text { NGA; Biermasz, } \\
\text { NR. }\end{array}$ & $\begin{array}{l}\text { The development and } \\
\text { validation of the } \\
\text { Leiden Bother and } \\
\text { Needs Questionnaire } \\
\text { for patients with } \\
\text { pituitary disease: } \\
\text { the LBNQ-Pituitary } \\
\text { (2016) }\end{array}$ & $\begin{array}{c}\text { Desenvolver e validar } \\
\text { questionário específico } \\
\text { para pacientes com } \\
\text { doença hipofisária que } \\
\text { incorpora pacientes com } \\
\text { incômodo relacionado a } \\
\text { doença e suas } \\
\text { necessidades de apoio. }\end{array}$ & $\begin{array}{l}\text { Questionário com pontuação. } \\
\text { O questionário levou a um } \\
\text { melhor entendimento dos } \\
\text { profissionais que cuidam de } \\
\text { pacientes com doenças } \\
\text { hipofisárias, observando suas } \\
\text { maiores queixas, dificuldades, } \\
\text { e suas necessidades de apoio. } \\
\text { Atentar-se para isso leva a } \\
\text { uma melhora na qualidade de } \\
\text { vida do paciente. }\end{array}$ \\
\hline 10 & $\begin{array}{c}\text { Teixeira, CR; } \\
\text { Scortegagna, } \\
\text { SA; Pasian, SR; } \\
\text { Portella, MR. }\end{array}$ & $\begin{array}{c}\text { Bem-estar subjetivo } \\
\text { de longevos } \\
\text { institucionalizados e } \\
\text { não } \\
\text { institucionalizados por } \\
\text { meio do Pfister (2019) }\end{array}$ & $\begin{array}{c}\text { Este estudo investigou o } \\
\text { Bem-Estar Subjetivo } \\
\text { (BES) de longevos } \\
\text { institucionalizados e não } \\
\text { institucionalizados por } \\
\text { meio do Teste das } \\
\text { Pirâmides Coloridas de }\end{array}$ & $\begin{array}{l}\text { Escalas. O estudo dos grupos } \\
\text { evidenciou sinais de vivências } \\
\text { depressivas (GDS-15). Os } \\
\text { achados contribuem para } \\
\text { compreensão da } \\
\text { psicodinâmica na velhice e } \\
\text { futuro planejamento de }\end{array}$ \\
\hline
\end{tabular}




\begin{tabular}{|c|c|c|c|c|}
\hline & & & Pfister (TPC). & $\begin{array}{l}\text { estratégias e intervenções que } \\
\text { fomentem o bem-estar } \\
\text { subjetivo dos longevos. }\end{array}$ \\
\hline 11 & $\begin{array}{c}\text { Swagerman, SC; } \\
\text { Geus, EJC; Kan, } \\
\text { KJ; Bergen, EV; } \\
\text { Nieuwboer, HA; } \\
\text { Gur, RE; Gur, } \\
\text { RC; Koenis, } \\
\text { MMG; Pol, } \\
\text { HEH; } \\
\text { Boomsma, DI. }\end{array}$ & $\begin{array}{l}\text { The Computerized } \\
\text { Neurocognitive } \\
\text { Battery: Validation, } \\
\text { Aging Effects, and } \\
\text { Heritability Across } \\
\text { Cognitive Domains } \\
\text { (2016) }\end{array}$ & $\begin{array}{c}\text { Estimar a validade e } \\
\text { confiabilidade da } \\
\text { tradução holandesa da } \\
\text { bateria, segundo } \\
\text { investigar efeitos da idade } \\
\text { em vários domínios } \\
\text { cognitivos, e terceiro para } \\
\text { estimar como essas } \\
\text { habilidades cognitivas } \\
\text { são influenciados por } \\
\text { fatores ambientais e } \\
\text { genéticos. }\end{array}$ & $\begin{array}{c}\text { Bateria Neurocognitiva } \\
\text { Computadorizada. CNB é um } \\
\text { instrumento confiável e válido } \\
\text { na população holandesa, com } \\
\text { pontuação comparáveis aos } \\
\text { estudos nos Estados Unidos. } \\
\text { A CNB é um valioso } \\
\text { instrumento não só para } \\
\text { pesquisa, mas também para } \\
\text { fins clínicos, exames clínicos } \\
\text { que incluem } \\
\text { neuropsicológicos } \\
\text { regularmente inteligência e } \\
\text { testes cognitivos, porque a } \\
\text { disfunção cognitiva é muitas } \\
\text { vezes uma característica de } \\
\text { distúrbios psiquiátricos. }\end{array}$ \\
\hline 12 & $\begin{array}{c}\text { César, KG; } \\
\text { Yassuda, MS; } \\
\text { Porto, FHG; } \\
\text { Brucki, SMD; } \\
\text { Nitrini, R. }\end{array}$ & $\begin{array}{c}\text { Addenbrooke's } \\
\text { cognitive } \\
\text { examination-revised: } \\
\text { normative and } \\
\text { accuracy data for } \\
\text { seniors with } \\
\text { heterogeneous } \\
\text { educational level in } \\
\text { Brazil (2017) }\end{array}$ & $\begin{array}{c}\text { Fornecer normas de } \\
\text { ACE-R para idosos com } \\
\text { menor escolaridade, } \\
\text { incluindo analfabetos. } \\
\text { Um objetivo adicional foi } \\
\text { examinar a precisão do } \\
\text { ACE-R para detectar } \\
\text { demência e } \\
\text { comprometimento } \\
\text { cognitivo sem demência. }\end{array}$ & $\begin{array}{c}\text { Exame Cognitivo de } \\
\text { Addenbrooke Revisado } \\
\text { (ACE-R). } \\
\text { Os resultados atuais } \\
\text { confirmam a alta precisão do } \\
\text { instrumento no diagnóstico de } \\
\text { demência, mesmo entre os } \\
\text { idosos com baixa } \\
\text { escolaridade. O ACE-R } \\
\text { também provou ser um } \\
\text { instrumento apropriado para o } \\
\text { diagnóstico de } \\
\text { comprometimento cognitivo } \\
\text { sem demência. }\end{array}$ \\
\hline
\end{tabular}


13

Tam, BWH; Lo,

DRT; Seah,

DWH; Lee, JX;

Foo, ZFY; Poh,

ZYY; Thong,

FXJ; Sim, SKY;

Chee, CS.
Developing and

validating a localised, self-training mindfulness programme for older Singaporean adults: effects on cognitive functioning and implications for healthcare (2017)

Rodrigues, JC;

Muller, JL;

Esteves, C;

Fonseca, RP;

Parente,

MAMP; Salles, JF.
Efeito de idade e escolaridade no instrumento de avaliação neuropsicológica breve NEUPSILIN (2018)

\section{Desenvolver um}

programa de mindfulness (atenção plena), de autotreinamento, guiado por profissionais especializados e testes de usabilidade, para adultos mais velhos de Cingapura para tornar a atenção plena mais disponível para aqueles que, de outra forma, não teriam acesso ao treinamento presencial tradicional em sala de aula devido a várias restrições.

Investigação de efeitos de idade e escolaridade e suas interações no desempenho de adultos no Instrumento de Avaliação

Neuropsicológica Breve NEUPSILIN, que avalia orientação têmporoespacial, atenção, percepção, memória, linguagem, calculias, praxias e funções executivas.
Programa de mindfulness (atenção plena), de autotreinamento. Houve uma melhoria geral na atenção e nas funções executivas dos participantes. No entanto, a melhora no grupo de treinamento não foi significativamente maior do que no grupo controle. Não obstante, a melhoria geral da atenção plena estava alinhada com o objetivo do treinamento.
Questionário de avaliação. Em que os resultados destacam que o envelhecimento e a escolaridade impactam de

forma heterogênea nas funções cognitivas, assim como os testes

neuropsicológicos brasileiros devem sempre considerar a influência dessas variáveis para produzir seus dados normativos.

\section{A tecnologia cuidativo-educacional e a enfermagem}

\begin{tabular}{|c|c|c|c|}
\hline & O cultivo do bem & Realizar avaliação & Aplicação das tecnologias \\
Goes, TM; & viver das pessoas & diagnostica de condições & cuidativo-educacionais em \\
Polaro, SHI; & idosas e tecnologia & de vida e saúde dos & saúde no envelhecimento. \\
Gonçalves, & cuidativo-educacional & idosos convivendo em & O estudo apresentou \\
LHT. & da enfermagem & família e comunidade, & resultados benéficos aos \\
& $(2016)$ & usuários de uma Unidade & idosos que participaram, bem \\
\hline
\end{tabular}




\begin{tabular}{|c|c|c|c|c|}
\hline & & & $\begin{array}{l}\text { Básica de Saúde - UBS e, } \\
\text { testar o desenvolvimento } \\
\text { de uma tecnologia } \\
\text { cuidativo-educacional aos } \\
\text { mesmos idosos, } \\
\text { considerando o } \\
\text { atendimento das } \\
\text { necessidades detectadas } \\
\text { na avaliação diagnostica. }\end{array}$ & $\begin{array}{c}\text { como à enfermagem, pela } \\
\text { possibilidade de empreender } \\
\text { ações cuidativo-educacionais } \\
\text { inovadas a favor do } \\
\text { autocuidado no } \\
\text { envelhecimento. }\end{array}$ \\
\hline 16 & $\begin{array}{c}\text { Silva, LMC; } \\
\text { Surniche, CA; } \\
\text { Sicsú, NA; } \\
\text { Mitano, F; } \\
\text { Nogueira, JÁ; } \\
\text { Santos, CB et al. }\end{array}$ & $\begin{array}{c}\text { Elaboração e } \\
\text { validação semântica } \\
\text { de um instrumento de } \\
\text { avaliação da } \\
\text { transferência do } \\
\text { tratamento } \\
\text { diretamente observado } \\
\text { como política de } \\
\text { controle da } \\
\text { tuberculose (2015) }\end{array}$ & $\begin{array}{c}\text { Elaborar e validar } \\
\text { semanticamente um } \\
\text { instrumento de avaliação } \\
\text { da transferência do } \\
\text { tratamento diretamente } \\
\text { observado (TDO) como } \\
\text { política de controle da } \\
\text { tuberculose segundo a } \\
\text { experiência de } \\
\text { profissionais de saúde de } \\
\text { nível médio e superior. }\end{array}$ & $\begin{array}{c}\text { Instrumento de avaliação da } \\
\text { transferência do tratamento } \\
\text { diretamente observado (TDO) } \\
\text { como política de controle da } \\
\text { tuberculose. } \\
\text { O instrumento foi considerado } \\
\text { importante para a prática } \\
\text { profissional, onde o processo } \\
\text { e as respostas geraram } \\
\text { modificações na estrutura e no } \\
\text { conteúdo resultando em um } \\
\text { instrumento elaborado e } \\
\text { validado semanticamente. }\end{array}$ \\
\hline 1 & $\begin{array}{l}\text { Simon, CM; } \\
\text { Klein, DW; } \\
\text { Schartz, HA. }\end{array}$ & $\begin{array}{l}\text { Interactive multimedia } \\
\text { consent for } \\
\text { biobanking: a } \\
\text { randomized trial } \\
(2016)\end{array}$ & $\begin{array}{l}\text { O objetivo deste estudo } \\
\text { foi testar a eficácia } \\
\text { separada da interatividade } \\
\text { cara a cara e por meio de } \\
\text { multimídia para melhorar } \\
\text { o entendimento e } \\
\text { confiança do participante } \\
\text { quanto a compreensão do } \\
\text { consentimento } \\
\text { informado. }\end{array}$ & $\begin{array}{l}\text { Interatividade cara a cara e } \\
\text { por meio de multimídia e } \\
\text { interatividade permitem uma } \\
\text { maior interação entre o } \\
\text { participante e pesquisador, no } \\
\text { entanto, o método permite um } \\
\text { feedback mais elaborado, } \\
\text { embora não seja imediato. Os } \\
\text { resultados deste estudo } \\
\text { sugerem que o método é tão } \\
\text { bom quanto, e talvez melhore } \\
\text { que, o processo de } \\
\text { consentimento padrão de } \\
\text { BioBanco na promoção da } \\
\text { compreensão das }\end{array}$ \\
\hline
\end{tabular}




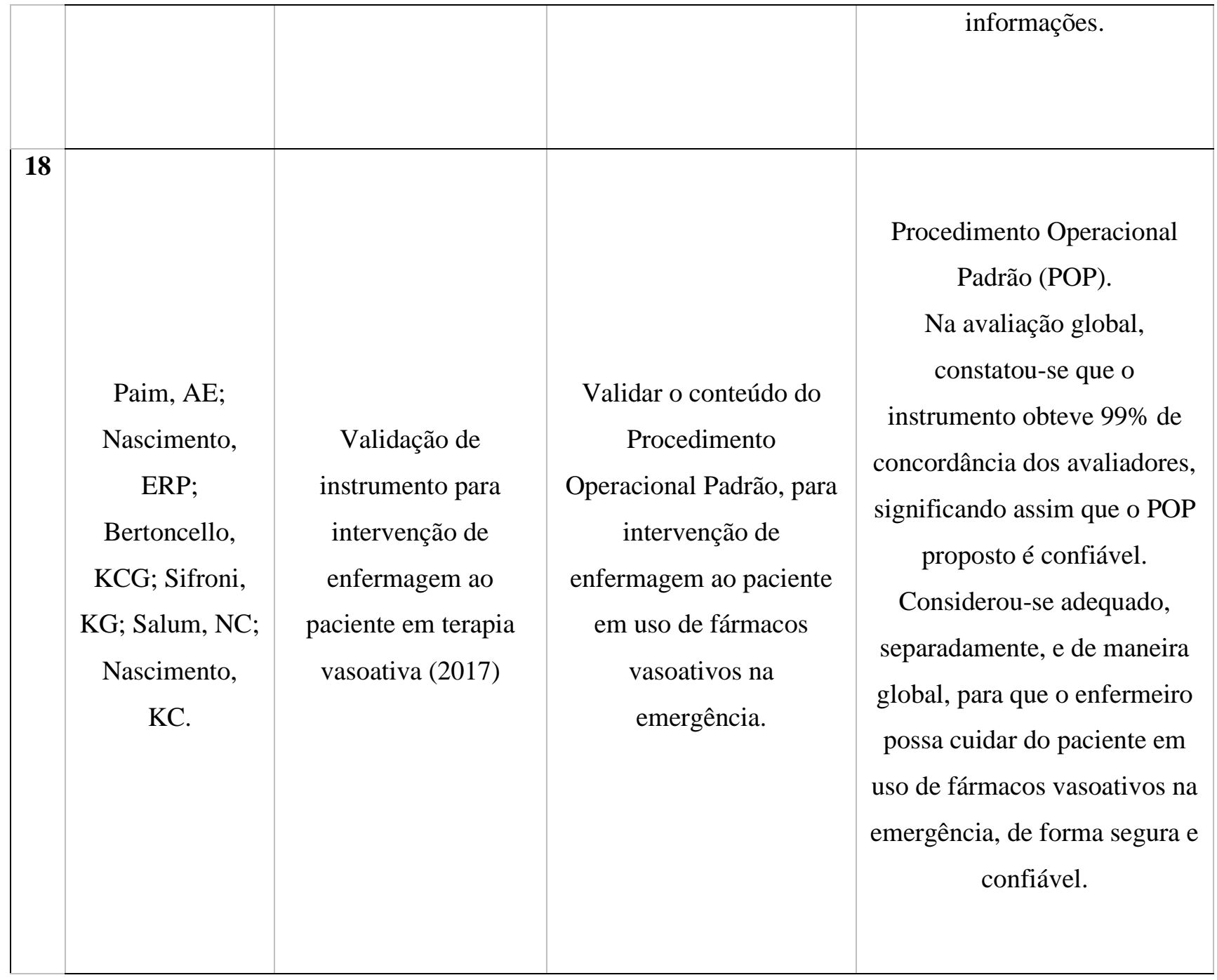

Fonte: Os autores.

DISCUSSÃO

\section{AÇÕES CUIDATIVAS EDUCACIONAIS}

Diversos fatores estão inseridos como percepções e subjetividades inerentes ao ser humano e não difere quando nos referimos à terceira idade, fase da vida a qual necessita de olhares atentos, uma vez que o envelhecimento ocasiona um natural declínio no idoso, que pode interferir na qualidade de vida, afetando-o nos domínios cognitivos, comportamentais, físicos e comunicativos, determinando a ampliação de limitações ${ }^{(9)}$.
Logo, é inegável que a existência de comprometimentos possam surgir com o decorrer do tempo e sejam contornados, trabalhados e amenizados, tornando possível o convívio independente e autônomo do idoso empregando programas de promoção à saúde, como a prática de exercícios físicos, hábitos alimentares saudáveis e atividades com estímulo da memória que auxiliem na administração de fatos negativos e no desfrutar de emoções positivas, nutrindo a confiança em si mesmo e no outro, através de experiências de domínio e relações 
recompensadoras que promovam e fortaleçam hábitos saudáveis e autoconfiança ${ }^{(10)}$.

A utilização de ferramentas que viabilizam ações cuidativas educacionais se configura como um instrumento de trabalho e também de lazer, inovando a aquisição e partilha da informação, ou seja, a comunicação torna-se essencial para manter a personalidade, um senso de identidade e conexão. Todavia cada indivíduo possui suas especificidades e particularidades vivenciadas o que pode vir a influenciar no modo de analisar e construir interação ou estratégia de ação por parte de um profissional ${ }^{(11)}$.

Essas ferramentas ajudam a desmistificar concepções e paradigmas ultrapassados da sociedade, que rotula o idoso como ser incapaz de aprender a viver com independência e autonomia, desempenhando e executando suas atividades de vida diária. Vislumbrando assim, uma alternativa viável, fácil e prática para se trabalhar ações cuidativas educacionais em saúde na terceira idade, como atividades recreativas populares onde os participantes, motivam seus sentimentos e memórias positivas e interagem socialmente $^{(12)}$.

Atividades educativas que fazem uso de linguagem clara, considerando a instrução do idoso e não o infantilizando, são mais eficazes. O uso de tecnologias como vídeos, iPad ou livros educativos viabilizam a compreensão e detecção de sintomas, auxiliando na tomada de decisão, no que tange a procura por serviços de atenção a saúde. Atividades recreativas estimulam o domínio cognitivo e o humor, favorecendo maior interação social que possibilita atividade corporal e mental ${ }^{(13-14)}$.

Desta forma, para se ter uma boa eficácia no uso de tecnologias educacionais com idosos, é necessário conhecimento/preparo não apenas sobre a tecnologia, mas também sobre o processo de envelhecimento humano, as funções físicas e mentais, bem como o cuidado que esta faixa etária pode necessitar.

Para isso, há necessidade de um bom vínculo com o idoso, aliado com uma preparação e orientação de qualidade do cuidador e também dos familiares, que muitas vezes são os cuidadores do idoso. Essa relação estimula a percepção do cuidador nos seguintes aspectos: conhecer a limitação do idoso e estímulo à autonomia, permitindo seu crescimento pessoal e mantêm um ambiente propício para a sua educação. Além disso, o preparo técnico do profissional é essencial para a oferta do cuidado qualificado e efetivo (15).

Para um essencial processo comunicacional com o idoso, os profissionais precisam adotar mecanismos interacionais que estimule a participação do idoso na interação, considerando as alterações individuais advinda do processo de envelhecimento humano. Logo, pode adotar estratégias de escrita, leitura, formas 
geométricas, com a finalidade de desenvolver o funcionamento cognitivo e habilidades psicomotoras ${ }^{(16)}$.

No entanto, tais tipos de estratégias, necessitam serem validadas quanto a sua eficácia e alcance dos objetivos propostos. De forma geral, a validação é feita a partir de uma análise feita por juízes, a fim de verificar o grau de concordância em relação à adequação das tarefas do treino às habilidades pretendidas ${ }^{(17)}$.

Devendo-se especificar o modelo avaliativo, no caso o questionário do avaliador e a perspectiva teórica, explicando a definição metodológica da área durante a construção e testagem dos programas da tecnologia de modo a fornecer evidências robustas de validade das tarefas elaboradas (17).

Assim, os artigos agrupados nesta categoria (1-8) demonstraram que a abordagem avaliativa auxilia na melhor compreensão de declínios inerentes ao ser idoso e que a implementação de programas de promoção à saúde melhora a vida e saúde das pessoas idosas, que se apresentam capazes de descobrir, assimilar e se transformar. Integrando assim o fazer, pensar e agir da pessoa idosa, na tentativa de empoderá-los a adoção de mudanças do estilo de vida.

Sendo esta categoria apresentou tecnologias que variam, de um programa de computador, avaliado por instrumentos psicológicos antes e após o tratamento (2);
Uma plataforma que integra o rastreamento da cabeça e tarefas cognitivas às atividades de equilíbrio baseada em jogos de computador (1); Um programa de intervenção por meio de desenhos, para verificar a capacidade basal e de realizar as tarefas sob diferentes domínios (7), além de outro de recreação popular, voltado para a melhora da função cognitiva (6).

Além disso, também se observou nos artigos (3-5) respectivamente, a interação comunicativa, por meio de linguagem simples e não infantilizada; $\mathrm{O}$ uso de vídeos educativos, iPads e livretos; E o desenvolvimento de material impresso e digital para orientação e informação do cuidado com o idoso (8).

INSTRUMENTOS AVALIATIVOS QUE AUXILIAM NA COMPREENSÃO DOS DECLÍNIOS DA TERCEIRA IDADE

A utilização de instrumentos avaliativos contribui positivamente na identificação da condição de saúde do idoso para os profissionais de saúde. Um dos métodos mais utilizados são os questionários, por serem de fácil aplicabilidade e baixo custo, no entanto ainda há deficiência nesses instrumentos quanto a multidimensionalidade frente a pessoa idosa, sendo observada frequentemente em países em desenvolvimento, por possuírem características diferenciadas. Para obter melhores resultados na coleta de informações, 
tais instrumentos devem conhecer o indivíduo idoso de forma eficaz e generalizada ${ }^{(18)}$.

Para que o idoso possa ter uma boa condição de saúde, é necessário entender quais alterações implicam no seu bem-estar ${ }^{(19)}$. Desse modo, a partir do entendimento da condição de saúde da pessoa idosa, através das informações colhidas dos instrumentos, é possível que o profissional implemente medidas de intervenção e promoção de saúde de maneira mais eficiente, visando sempre a qualidade de vida do idoso.

A partir da compreensão de que a pessoa idosa não deve ser vista somente com olhar clínico, por outros e por si próprio, é de suma importância o apoio familiar, bem como a percepção subjetiva de saúde e satisfação de vida, sendo possível a vivência positiva dessa fase, em que pensando-se em termos de prevenção e promoção da saúde há uma consequente influência no bem-estar físico e mental do idoso ${ }^{(19)}$.

Com os declínios da terceira idade é preciso buscar a melhor maneira de trabalhar com esses indivíduos, visando sempre a qualidade de vida. A inclusão da pessoa idosa no convívio familiar e social é de grande importância ${ }^{(20)}$. Dessa forma, é possível haver o estreitamento de vínculo com profissionais da saúde e cuidadores.

O conjunto de artigos apresentados nesta categoria (09-11), mostra a importância de ofertar assistência tendo por base a utilização de instrumentos avaliativos que contribuem na detecção fidedigna da real condição de saúde do idoso, favorecendo a implantação de medidas de intervenção e promoção à saúde, além de viabilizar o estreitamento de vínculo entre os idosos e os profissionais de saúde, como também os cuidadores.

Nesta categoria foram encontradas durante as análises, um questionário desenvolvido especificamente para pacientes com doenças hipofisárias (9); Um estudo que investiga o Bem-Estar Subjetivo (BES) de idosos institucionalizados e não institucionalizados através do Teste de Pirâmides Coloridas de Pfister (TPC) (10).

Além disso, foi identificado um outro estudo referente a cognição, o Exame Cognitivo de Addenbrooke Revisado (ACE$\mathrm{R})$, que por sua vez mede o nível cognitivo de idosos com baixa escolaridade, bem como os que não são alfabetizados. Somando a isso, o estudo ainda buscou detectar demência e comprometimento cognitivo sem demência (11)

A TECNOLOGIA CUIDATIVO EDUCACIONAL E A ENFERMAGEM

Atualmente, a enfermagem realiza um papel fundamental quando se fala em cuidado $\mathrm{e}$, inerente a isso, tem-se a necessidade de estar atualizando constantemente os conhecimentos e técnicas dos profissionais, tornando-os aptos a trabalhar com novos meios que permitam o melhor cuidado. A 
enfermagem vem evoluindo enquanto ciência e profissão, e isso se deve aos novos meios tecnológicos, que emanam a necessidade de adaptação e conhecimento de ferramentas auxiliadoras para o cuidado.

Com base nas análises dos estudos pesquisados, é visível a necessidade do conhecimento sobre a utilização de tecnologias educacionais voltadas a saúde da pessoa idosa, em que devido ao intenso processo de transição demográfica, um plano de cuidado direcionado as especificidades do idoso, possa ser viabilizado pelo uso das tecnologias cuidativo educacionais.

Portanto, denota-se a importância de estratégias diversificadas e práticas transformadoras como a utilização das tecnologias cuidativo-educacionais em saúde (TCESs), que são ferramentas importantes no âmbito da assistência de enfermagem, pois permite aproveitar o acompanhamento do idoso à unidade de saúde para aplicação da tecnologia, promovendo a educação em saúde e estímulo ao auto cuidado ${ }^{(21-22)}$.

Assim, na utilização de uma TCES deve-se ter um planejamento e, além disso, saber maneja-lo como um instrumento da prática pelo profissional enfermeiro. Portanto, diante da necessidade de que o instrumento elaborado tenha fidelidade para a sua aplicação, foi elaborada uma questão de pesquisa chamado de "Procedimento Operacional Padrão" (POP), visando a orientação das intervenções junto ao paciente alvo e objetivando a validade do conteúdo de modo a se tornar uma ferramenta segura no cuidado ${ }^{(23)}$.

Sendo assim, o "Tratamento

Diretamente Observado" (TDO), o instrumento utilizado como política, estaria voltada para o controle da tuberculose na qual três dimensões se apresentam intrinsicamente ligadas, sendo: a informação recebida pelos profissionais responsáveis pela aplicação da política/ação/instrumento; o conhecimento dos mesmos acerca da política de implementação, bem como a assimilação das informações recebidas; e a inovação, ou modo de implementação e execução propriamente dito, dessa nova política/ação ${ }^{(24)}$.

Esta categoria (12-15) apresentou como o uso de tecnologias viabiliza o cuidar de diversos profissionais da saúde, dentre eles os enfermeiros, que a partir disso, podem empreender ações cuidativo-educacionais que permitem um cuidar seguro e eficaz.

Sendo as tecnologias desta categoria instrumentos de avaliação escrita (12-14), usados para diversas funções. Já o artigo (15) utiliza recursos de multimídia para avaliar a diferença de compreensão de informações entre o mesmo e a cara a cara.

\section{CONCLUSÃO}

Identificou-se, nesta revisão integrativa, que as tecnologias educacionais desenvolvidas e/ou validades para o cuidado com a pessoa idosa foram diversas, com predominância para plataformas digitais como 
vídeos educativos, iPads e livretos, além do uso de instrumentos avaliativos que viabilizam o cuidar e estreitamento de vínculo com o idoso, família e comunidade, sendo os enfermeiros os profissionais que mais utilizam as tecnologias.

O tema mais abordado pelos estudos foi a utilização de tecnologias voltadas a avaliação e estímulo cognitivo, variando entre programas de computador de rastreio, análise do decréscimo cognitivo, meios de orientação ou prática intervencionista de atividades voltadas aos idosos. Apontam-se como lacunas do conhecimento a falta artigos abordando a validação de TECS direcionadas ao cuidado cognitivo do idoso.

Sugere-se o desenvolvimento de outros estudos que desenvolvam e validem tecnologias educacionais para pessoas idosas capazes de estimular os domínios da cognição, humor, mobilidade e comunicação, visando o envelhecimento ativo e saudável capaz de viabilizar o auto cuidado, auto valorização, autonomia e independência do idoso.

\section{REFERÊNCIAS:}

1 - Faller JW, Teston EF, Marcon SS. Estrutura conceptual do envelhecimento em diferentes etnias. Rev. Gaúcha Enferm. 2018; 39(66144).

2- Instituto Brasileiro de Geografia e Estatística. Contagem populacional: censo demográfico 2010. Brasília, DF.
3 - Rocha MRC, Araújo, JC, Farias TPF, Barros AMS. Avaliação Funcional e Cognitiva do Idoso. In: Congresso Internacional de Enfermagem. 2017; 1(1).

4 - Bezerra, PK, Rodrigues KA, Felix KD, Sotero RC, Ferreira AP. Déficit cognitivo: proposição de cartilha para atenção ao idoso. Revista Brasileira de Pesquisa em Ciências e Saúde. 2016; 3(1): 1-10.

5 - Moreira, APA Sabóia VM, Camacho ACLF, Daher D V, Teixeira E. Jogo educativo de administração de medicamentos: um estudo de validação. Rev. Bras. Enferm. 2014 Ago; 67(4): 528-34.

6- Brasil. Ministério da Educação. Secretaria de Educação Básica. Guia de Tecnologias Educacionais da Educação Integral e Integrada e da Articulação da Escola com seu Território 2013/MEC. Organização Paulo Blauth Menezes. Brasília; 2013.

7 - Gadelha MMT, Andrade ME, Silva JMA, Bezerra ICB, Carmo, AP, Fernandes MC. Tecnologias educativas no processo formativo: discurso dos acadêmicos de enfermagem. Rev. enferm. UFPE online. 2019; 13(1): 155-161.

8 - Mendes KDS, Silveira, RCCP, Galvão, CM. Revisão integrativa: método de pesquisa para a incorporação de evidências na saúde e na enfermagem. Texto contexto - enferm. 2018 Dez; 17(4): 758-64.

9 - Santos ALS, Santos AIPSS, Lourenço NLR Souza MO, Teixeira VPG. A importância do uso de tecnologias no desenvolvimento cognitivo dos idosos. GEP NEWS. Jan/Mar 2018; 1(1): 20-4.

10 - Caprara MG, Fernández-Ballesteros R, Alessandri, G. Promoting aging well: evaluation of vital-aging-multimedia program in Madrid, Spain. Health promotion international. 2016; 31(3): 515-22.

11 - Williams KN, Perkhounkova Y, Herman $\mathrm{R}$, Bossen A. A communication intervention 
to reduce resistiveness in dementia care: A cluster randomized controlled trial. The Gerontologist. 2017; 57(4):707-18.

12 - Li DM, Li, XX. The effect of folk recreation program in improving symptoms: A study of Chinese elder dementia patients. International journal of geriatric psychiatry. 2017 Ago; 32(8): 901-08.

13 - Dallimore RK, Asinas-Tan ML, Chan D, Hussain S, Willet $\mathrm{C}$, et al. A randomised, double-blinded clinical study on the efficacy of multimedia presentation using an iPad for patient education of postoperative hip surgery patients in a public hospital in Singapore. Singapore Medical Journal. 2017; 58(9): 562.

14 - Denny MC, Vahidy F, Vu KYT, Sharrief, Savitz SI. Video-based educational intervention associated with improved stroke literacy, self-efficacy, and patient satisfaction. PloS one. 2017 Mar 23; 12(3): 0171952.

15 - Cardoso RSS, Sá SPC, Domingos AM, Sabóia VM, Maia TN, Padilha JMFO, et al. Tecnologia educacional: um instrumento dinamizador do cuidado com idosos. Revista Brasileira de Enfermagem. 2018; 71(2): 78692.

16 - Szturm T, Sakhalkar V, Boreskie S, Marotta JJ, Wu C, Kanitkar A. Integrated testing of standing balance and cognition: Test-retest reliability and construct validity. Gait \& posture. 2015 ; 41(1): 146-52.

17 - Golino, MTS Schelini PW, Golino HF, Pereira BLS, Felix LM. Investigando Evidências de Validade de Conteúdo e Estrutural em Tarefas de um Treino Cognitivo para Idosos. Avaliação Psicológica. 2017; 16(3): 278-92.

18 - Pedreira RBS, Rocha SV, Santos CA, Vasconcelos LRC, Reis MC. Validade de conteúdo do Instrumento de Avaliação da Saúde do Idoso. Einstein. 2016; 14(2):158-77.

19 - Nakano TC, Machado, WL, Abreu, ICC. Relações entre estilos de pensar e criar, bem- estar, saúde percebida e estresse na terceira idade. Psico-USF. 2019 Set; 24(3): 555-68.

20 - Bittencourt AM, Machado LFO, Almeida MBde, Pires DR. Software: recurso terapêutico ocupacional para estimulação cognitiva do idoso. Estudos Interdisciplinares sobre o Envelhecimento. 2017; 22(10): 31-49.

21 - Goes TM, Polaro SHI, Gonçalves LHT. Cultivo do bem viver das pessoas idosas e tecnologia cuidativo-educacional de Enfermagem. Enfermagem em Foco. 2016; 7(2): 47-51.

22 - Simon CM, Klein DW, Schartz HA. Interactive multimedia consent for biobanking: a randomized trial. Genetics in Medicine. 2016; 18(1): 57-64.

23 - Paim, AE, Nascimento ERP, Bertoncello KCG, Sifroni KG, Salum NC, Nascimento KC. Validação de instrumento para intervenção de enfermagem ao paciente em terapia vasoativa. Revista Brasileira de Enfermagem. 2017; 70(3): 453-60.

24 - Silva LMC, Surniche CA, Sicsú AN, Mitano F, Nogueira JdeA, Santos, CB. Elaboração e validação semântica de um instrumento de avaliação da transferência do tratamento diretamente observado como política de controle da tuberculose. Revista Panamericana de Salud Pública. 2015; 38(2): 129-35.

25 - Andela CD, Scharloo M, Ramondt S, Jitske $\mathrm{T}$, Olga $\mathrm{H}$, Sofia $\mathrm{L}$ et al. The development and validation of the Leiden Bother and Needs Questionnaire for patients with pituitary disease: the LBNQ-Pituitary. Pituitary. 2016; 19(3): 293-302.

26 - César KG, Yassuda MS, Porto FHG, Brucki SMD, Nitrini R. Addenbrooke's cognitive examination-revised: normative and accuracy data for seniors with heterogeneous educational level in Brazil. International psychogeriatrics. 2017; 29(8): 1345-53. 
27 - Teixeira CR, Scortegagna SA, Portella MR, Pasian SR. Bem-estar subjetivo de longevos institucionalizados e não institucionalizados por meio do Pfister. Avaliaçao Psicologica: Interamerican Journal of Psychological Assessment. 2019; 18(1): 86-95.

28 - Rodrigues JC, Muller JL, Esteves C, Fonseca RP, Parente MAMP, Salles JF de. Efeito de idade e escolaridade no instrumento de avaliação Neuropsicológica Breve NEUPSILIN. Psico-USF. 2018; 23(2): 31932.

29 - Swagerman SC et al. The Computerized Neurocognitive Battery: Validation, aging effects, and heritability across cognitive domains. Neuropsychology. 2016; 30(1): 53.

30 - Tam BWH, Lo DRT, Seah DWH, Lee JX, Foo ZFY, Poh ZYY et al. Developing and validating a localised, self-training mindfulness programme for older Singaporean adults: effects on cognitive functioning and implications for healthcare. Singapore Medical Journal. 2017; 58(3): 126. 2017.

Submissão: $2020-12-08$

Aprovado: 2021-03-25 